\title{
Eisen hilft bei Herzschwäche
}

- Eisenmangel verursacht nicht nur Anämie, sondern kann auch zu verminderter Leistungsfähigkeit und zu neurologischen Komplikationen führen. Denn Eisen ist außer für die Blutbildung auch für die zelluläre Energiegewinnung und die Neurotransmittersynthese essenziell. Dass das Auffüllen der Eisenspeicher auch bei Eisenmangel ohne Anämie sinnvoll sein kann, wurde in der FAIR-HF-Studie bei Herzinsuffizienzpatienten eindeutig nachgewiesen.

Im Rahmen der Studie wurden 459 Patienten mit systolischer Herzinsuffizienz und Eisenmangel mit i.v. Eisencarboxymaltose (Ferinject ${ }^{\circledR}$ ) oder Kochsalzlösung behandelt. Die Eisenmangelkorrektur führte auch bei nicht anämischen Patienten bereits nach vier Wochen zur hochsignifikanten Verbesserung von Leistungsfähigkeit und Lebensqualität.

Auch Patienten mit chronisch entzündlicher Darmerkrankung, chronischer Niereninsuffizienz oder Krebs leiden häufig an funktionellem Eisenmangel.
Sie profitieren ebenfalls von der i.v. Applikation.

Eisencarboxymaltose ist indiziert zur Behandlung von Eisenmangelzuständen, wenn orale Eisenpräparate unwirksam sind oder nicht angewendet werden können. Mit dem Präparat gelingt es, einfach und langfristig die Eisenspeicher aufzufüllen. In kurzer Zeit lassen sich hohe Dosen Eisen (bis zu $1000 \mathrm{mg}$ in mindestens 15 Minuten) applizieren. Vorteile gegenüber herkömmlichen Eisenpräparaten sind die hohe Komplexstabilität und die gute Verträglichkeit. Die innovative Galenik verhindert die unkontrollierte Freisetzung von Eisen in der Blutbahn und schützt so vor den toxischen freien Eisenionen.

Ferinject ${ }^{\circledast}$ von Vifor gehört zu den Kandidaten für den diesjährigen Galenus von Pergamon-Preis. Mit dem Preis zeichnet die „Ärzte Zeitung“ herausragende Arzneimittelinnovationen aus. Die Preisverleihung erfolgt am 21. Oktober in Berlin. - $E B$

\section{Sinnvoll kombinieren gegen Typ-2-Diabetes Gewichtskontrolle ist auch Risikokontrolle}

- Der Therapieeinstieg mit Metformin bei Typ-2-Diabetes ist unumstritten. Aber was ist der geeignete Kombinationspartner unter den von der DDG gleichberechtigt gelisteten Zweitlinien-Antidiabetika? Hier gebe es für jeden Patienten andere Prioritäten, sagte Prof. Andreas Hamann, Bad Nauheim. Neben Kriterien wie der Hypoglykämieneigung, der Distanz zum $\mathrm{HbA}_{1 \mathrm{c}}$-Ziel und dem Vorliegen von Langzeit-Sicherheitsdaten wies Hamann eindringlich auf die Bedeutung der Gewichtsentwicklung unter der Therapie hin. Der positive Effekt einer antidiabetischen Therapie könne durch Adipositas verringert werden. „Das müssen wir uns vergegenwärtigen, wenn wir uns für Therapieformen entscheiden, die eine Gewichtszunahme fördern“, sagte Hamann.
In einer aktuellen Metaanalyse aus 27 kontrollierten Studien hat sich bestätigt, dass DPP-4-Hemmer und GLP-1-Analoga zusätzlich zur maximalen Metformintherapie auch hinsichtlich der Gewichtskontrolle gut abschneiden (Phung OJ et al. JAMA 2010). Dabei stellten sich die oralen DPP-4-Hemmer wie Sitagliptin (z.B. Xele$\mathrm{via}^{\circledR}$, in Fixkombination mit Metformin z. B. Velmetia ${ }^{\circledR}$ ) erneut als gewichtsneutral heraus. Für welchen der inkretinbasierten Wirkstoffe man sich letztlich entscheidet, hänge besonders von der bevorzugten Applikationsform ab, so Dr. Thorsten Siegmund, München.

Effektiv seien beide Therapien, so Siegmund. So habe eine aktuelle Studie, in der Metformin entweder mit Liraglutid oder Sitagliptin kombiniert wurde, nach
Thromboseprophylaxe mit NMH

Klick-System schützt vor Nadelstichverletzung

- Das niedermolekulare Heparin Dalteparin (Fragmin ${ }^{\circledR}$ P/Forte) ist in neuen Sicherheitsspritzen erhältlich. Sie verringern die Gefahr einer Nadelstichverletzung und reduzieren den „Spritzenmüll“. Neu an den TRBA-250-konformen Sicherheitsspritzen ist das „Klick-System“: Nach der Injektion drückt der Anwender die Schutzvorrichtung mit einer Hand gegen eine stabile Oberfläche und biegt die Nadel dadurch so weit um, bis sie in die Schutzvorrichtung einrastet (Abb.). Das Einrasten ist durch ein Klickgeräusch hörbar. Im Verletzungsgefahr gebannt. Vergleich zu passiven Sicherheitssystemen haben die neuen Spritzen auch den Vorteil eines geringeren Volumens, sodass Lagerung und Entsorgung weniger Platz beanspruchen. An der Injektionstechnik hat sich nichts geändert. Die bisherigen Fertigspritzen werden sukzessive aus dem Handel genommen.

- Quelle: Pressemitteilung Pfizer, 17.6.2010

26 Wochen für $100 \mathrm{mg} / \mathrm{d}$ Sitagliptin vs. $1,2 \mathrm{mg} / \mathrm{d}$ Liraglutid lediglich eine mittlere Differenz des $\mathrm{HbA}_{1 \mathrm{c}}$-Werts von etwa 0,3 $\%$-Punkten ergeben (Pratley RE et al. Lancet 2010). Der mediane Gewichtsverlust differierte um etwa $2 \mathrm{~kg}$ zugunsten des Inkretinanalogons. Allerdings klagte etwa ein Fünftel der Patienten mit Liraglutid über Übelkeit, so Siegmund. Auch die drei derzeit erhältlichen Gliptine seien von der $\mathrm{HbA}_{1 c}$-Effektivität ähnlich. Allerdings ist bislang nur Sitagliptin zur Monotherapie bei Metforminunverträglichkeit und in Dreifachkombinationen sowie mit Insulin zugelassen.

\footnotetext{
- Sarah Pampel

Symposium, DDG-Jahrestagung, Stuttgart, 12. Mai 2010 (Veranstalter: Berlin Chemie)
} 